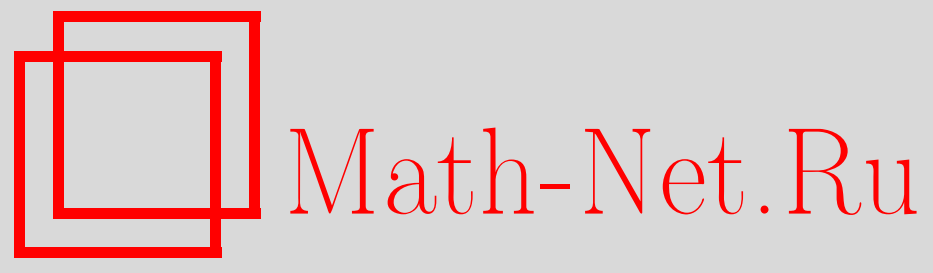

A. Yu. Samarin, Механическое движение специфической сплошной среды как физическая основа квантовой эволюции, Вестн. Сам. гос. техн. унта. Сер. Физ.-мат. науки, 2020, номер 1, 7-21

DOI: https://doi.org/10.14498/vsgtu1724

Использование Общероссийского математического портала MathNet.Ru подразумевает, что вы прочитали и согласны с пользовательским соглашением

http://www . mathnet.ru/rus/agreement

Параметры загрузки:

IP: 3.85 .183 .62

26 апреля 2023 г., 14:45:59

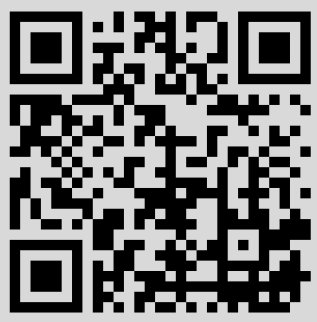




\title{
Differential Equations and Mathematical Physics
}

MSC: 81S40, 58D30

\section{Quantum evolution as a usual mechanical motion of peculiar continua}

\section{A. Yu. Samarin}

Samara State Technical University,

244, Molodogvardeyskaya st., Samara, 443100, Russian Federation.

\begin{abstract}
Quantum particles are considered as continuous media having peculiar properties. These properties are formulated so that all main quantum mechanics postulates can be strictly derived from them. A deterministic description of the process of position measurement is presented. The mechanism of occurrence of randomness in the measurement process is shown and the Born rule is derived. A realistic interpretation of the wave function as a component of a peculiar variable force acting on the apparatus is introduced, and the wave equation is derived from the continuity equation of the peculiar continuum. The deterministic view on the phenomena of the microcosm allows us to eliminate the limitations caused by the uncertainty principle and to describe dynamically those processes that cannot be considered using conventional quantum mechanics.
\end{abstract}

Keywords: deterministic quantum description, continuous medium, local realism principle, matter field, continuity equation, wave function realistic interpretation, Born rule, uncertainty principle.

Received: $21^{\text {st }}$ July, 2019 / Revised: $12^{\text {th }}$ October, $2019 /$

Accepted: $11^{\text {th }}$ November, $2019 /$ First online: $7^{\text {th }}$ February, 2020

\section{Research Article}

( ) (1) The content is published under the terms of the Creative Commons Attribution 4.0 International License (http://creativecommons.org/licenses/by/4.0/)

Please cite this article in press as:

S a marin A. Y u. Quantum evolution as a usual mechanical motion of peculiar continua, Vestn. Samar. Gos. Tekhn. Univ., Ser. Fiz.-Mat. Nauki [J. Samara State Tech. Univ., Ser. Phys. Math. Sci.], 2020, vol. 24, no. 1, pp. 7-21. doi: 10.14498/vsgtu1724.

Author's Details:

Alexey Yu. Samarin (1) https://orcid.org/0000-0001-7640-3875

Cand. Phys. \& Math. Sci.; Associate Professor; Dept. of General Physics and Physics of Oil and Gas Production; e-mail: samarin.ay@yahoo.com 


\section{Introduction}

The solving problem of the local realism [1] is a prerequisite for any fundamental microcosm theory. We sure that the nonrealistic attitude is unacceptable for any physical theory and the wave function has to be interpreted, in accordance with Srödinger's view [2] "... as giving somehow the density of the stuff of which the world is made" [3]. This means, inter alia, that any physical quantity is an attribute of a material object (as material objects quantum particles ${ }^{1}$ will be considered). Therefore the substance generating the wave function should be distributed in space. The type of this distribution, first of all, must satisfy the principle of locality. Then the simultaneous transformation of the wave function in the remote points in space, as the resulting of a local external effect on the quantum particle, forces us to suppose that there is no empty space between the parts of the substance. Thus, we have the continuum (hereinafter referred to as a physical continuum), which is not only a mathematical abstraction, but also a physical reality. In this sense, the concept of a material point (the term of individual particle will not be used here) of continuum mechanics should be taken literally as an element of the substance corresponding to a point in space, i.e. the set of the matter points that forms any particular body has the cardinality of the continuum. These material points can play the role of intermediaries in the instantaneous interaction between the material points that are remote in space. This assumption allows us to eliminate the violation of relativistic requirements [4] when interpreting the non-unitary processes (including the EPR paradox) while maintaining the principle of local realism.

\section{Attributes of the physical continuum}

The first property of the physical continuum is analogous to the classical one and consists in the fact that material points move in accordance with the principle of least action [5].

In addition, the physical continuum has some extraordinary properties. The second property is that there are no interaction forces between the material points forming the continuum of an elementary particle (there are no stresses within homogeneous physical continuum). ${ }^{2}$ This means that more than one of the material points can have the same position, i.e. the continuous medium can be a collection of continuous media. Taking into account the quantum superposition principle, it is logical to assume that these continuous media are formed, ultimately, by material fields. The fact of the interaction of remote in space particles in entangled states gives each of these fields its own physical reality, i.e., they exist even in those spatial regions where the wave function is zero.

Suppose that a quantum particle (hereinafter referred to as a quantum object or simply an object) is a homogeneous, inseparable object within quantum mechanics. This gives rise to the following specific features of the dynamics:

- the inertia property of each material point is determined by the total mass of the object, and not by the mass density, as in classical continuum mechanics

\footnotetext{
${ }^{1}$ We suppose that a quantum particle obeys the laws of conventional quantum mechanics. Immediately after measuring position it is described by the wave function in the form of a position delta-function, but it is not a point-like particle.

${ }^{2}$ This does not mean that there is no interaction between the material points at all. In the paper [6], the normalizing procedure required to describe the non-unitary process is considered as a mathematical image of a real physical process. Such a process is impossible without a peculiar interaction between material points.
} 
(the third property);

- any change in the measure of one of the material points subset in a local volume of space, instantly changes the measures of all the rest of other subsets in the whole space, which physically means that any local external effect on the quantum object result in the simultaneous transformation of the state of the entire continuous medium everywhere (this property have been considered in detail in [6]).

To introduce the spatial distribution of the physical continuum, define the measure $d M$ of the material point set occupying an infinitesimal volume $d V$ as

$$
d M=\frac{d Q}{Q}
$$

where $Q$ is any additive conservative quantity such as electric charge, gravitational mass of the quantum object etc; $d Q$ is the value of this quantity for a substance in the volume $d V$. Therefore, for the density of the measure, we have

$$
\rho(x, y, z)=\frac{d M}{d V}
$$

and for normalization condition

$$
\int_{-\infty}^{\infty} \int_{-\infty}^{\infty} \int_{-\infty}^{\infty} \rho(x, y, z) d x d y d z=1
$$

Taking into account the forth property of the physical continuum, the formal normalisation procedure (1) should be considered as a mathematical image of the real transformation of a continuous medium that accompanies any non-unitary processes in conventional quantum mechanics, such as the wave function collapse in the measurement process. In accordance with the definition the measure $M$ is an additive quantity having a positive real value.

Suppose, in accordance with the second property, that the continuous medium of any quantum object is formed by a set of the matter fields. Denote by $\xi_{1}=x(0)$, $\xi_{2}=y(0), \xi_{3}=z(0)$ are the referential coordinates of the material particle (hereinafter, the notation $\xi_{j}$ will be used as material coordinates (in this case index $j$ corresponds to the coordinate axes) whereas coordinate variables of the fields at time $t_{j}$ will be denoted by $x_{j}, y_{j}, z_{j}$. In accordance with the second property, material points can not uniquely be identified only by material coordinates (in general, their initial velocities are necessary), however, in the case of matter fields, material coordinates uniquely identify material points. We introduce the notion of the complex density of a measure (or simply a complex density) as a characteristic of a material point, such that

$$
\mu\left(\xi_{1}, \xi_{2}, \xi_{3}, \vec{v}_{0}, t\right)=\rho\left(\xi_{1}, \xi_{2}, \xi_{3}, \vec{v}_{0}, t\right) \exp \frac{i}{\hbar} S\left[x\left(\xi_{1}, \xi_{2}, \xi_{3}, \vec{v}_{0}, t\right), t, t_{0}\right],
$$

where the action $S\left[x\left(\xi_{1}, \xi_{2}, \xi_{3}, \vec{v}_{0}, t\right), t, t_{0}\right]$ is an attribute of the material point; material points move along the path $x\left(\xi_{1}, \xi_{2}, \xi_{3}, \vec{v}_{0}, t\right)$ corresponding to the principle of least action; $t_{0}$ is an initial time. For the one-dimensional motion, which will only be considered, we have

$$
\mu\left(\xi, v_{0}, t\right)=\rho\left(\xi, v_{0}, t\right) \exp \frac{i}{\hbar} S\left[x\left(\xi, v_{0}, t\right), t, t_{0}\right]
$$


And for a closed system, we obtain

$$
\mu(\xi, E, t)=\rho(\xi, E, t) \exp \frac{i}{\hbar} S\left[x(\xi, E, t), t, t_{0}\right] .
$$

where $E$ is the energy of the material point. If the material points of the matter field have the same moving direction and the same energy, then the continuity property conserves in time (see the next section for details). The function $\mu^{E, \alpha}(\xi, t)$ (the energy dependence and the motion direction are indicated by a superscript ${ }^{3}$ ) for a matter field can be rewritten in form of the field

$$
\mu^{E, \alpha}(x, t)=\int_{-\infty}^{\infty} \mu^{E, \alpha}(\xi, t) \delta\left(x-x^{E, \alpha}(\xi, t)\right) d \xi
$$

where $x^{E, \alpha}(\xi, t)$ is the path corresponding to the least action of the material point $\xi$ having the energy $E$ and the motion direction $\alpha$. Suppose that the complex density determines the summation law of the measure densities of those particles, that are in the same point in space in the form

$$
\mu(x, t)=\int_{0}^{\infty} \sum_{\alpha} g_{\alpha}(E) \mu_{\alpha}^{E}(x, t) d E
$$

$\mu(x, t)$ is a complex density of the measure. The complex weight $g_{\alpha}(E)$ of the fields forming a continuous medium is determined by the history of the formation of the continuum mechanical state. Thus, for the measure density, we obtain

$$
\rho(x, t)=|\mu(x, t)| .
$$

Since the measure (1) of objects conserves in the processes considering in quantum mechanics, for the measure density the continuity equation can be written. Dependence (2) allows us to write corresponding equation for the strength of a physical continuum $\mu$.

\section{The continuity equation}

First of all, it is necessary to determine the conditions under which a continuous medium remains continuous in time. In accordance with the second property of the physical continuum, this means that the mater fields that formed it at some initial time remain fields in the subsequent time. Suppose $F(x)$ is a stationary external force field. By $\xi_{1}=x_{1}\left(t_{0}\right)$ and $\xi_{2}=x_{2}\left(t_{0}\right)\left(x_{2}\left(t_{0}\right)>x_{1}\left(t_{0}\right)\right)$ denote the material coordinates (Lagrange variables) of two specified material points of the continuous medium. At the time $t$ their positions is expressed as

$$
\begin{aligned}
& x\left(\xi_{1}, t\right)=x_{1}\left(t_{0}\right)+\int_{t_{0}}^{t} v\left(\xi_{1}, \tau\right) d \tau, \\
& x\left(\xi_{2}, t\right)=x_{2}\left(t_{0}\right)+\int_{t_{0}}^{t} v\left(\xi_{2}, \tau\right) d \tau .
\end{aligned}
$$

\footnotetext{
${ }^{3}$ The direction index $\alpha$ has only two values "+" for the motion along the axis and "_" for the motion in the opposite direction. For brevity, it will be used only if necessary (in the cases of finite motion, when there are two continua moving in opposite directions).
} 
where $\tau$ is a time variable; $t$ denotes fixed points in time, such as the initial time $t_{0}$ and the current time $t ; v\left(\xi_{1}, \tau\right), v\left(\xi_{2}, \tau\right)$ are respectively the velocities of the first and the second material points. Then the distance between the considered points at an arbitrary time is determined by the expression

$$
\begin{aligned}
\Delta x= & \Delta x_{0}+\int_{t_{0}}^{t}\left(v\left(\xi_{2}, \tau\right)-v\left(\xi_{1}, \tau\right)\right) d \tau= \\
& =\Delta x_{0}+\int_{t_{0}}^{t}\left(v\left(\xi_{2}, t_{0}\right)-v\left(\xi_{1}, t_{0}\right)+\frac{1}{m} \int_{t_{0}}^{\tau}\left(F\left(\xi_{2}, \theta\right)-F\left(\xi_{1}, \theta\right)\right) d \theta\right) d \tau,
\end{aligned}
$$

where $\Delta x_{0}=x_{2}\left(t_{0}\right)-x_{1}\left(t_{0}\right) ; \theta$ is a time variable; $F\left(\xi_{1}, \theta\right), F\left(\xi_{2}, \theta\right)$ are the forces acting on the points. ${ }^{4}$ In order for the one-to-one correspondence between the material points $\xi$ and positions $x$ to take place all the time, it is necessary that

$$
\lim _{\Delta x_{0} \rightarrow 0} \Delta x=0
$$

Suppose, that the spatial interval $\Delta x_{0}$ is small. By $\varepsilon$ denote the time interval $\Delta x_{0} / v\left(\xi_{1}, t_{0}\right)$; by $\eta$ denote the spatial variable. Then

$$
\begin{aligned}
\frac{1}{m} \int_{t_{0}}^{\tau} F\left(\xi_{1}, \theta\right) d \tau \approx \frac{1}{m}\left(\int_{t_{0}+\varepsilon}^{\tau} F\left(\xi_{1}, \theta\right) d \theta+\varepsilon F\left(\xi_{1}, t_{0}\right)\right) & = \\
& =\frac{1}{m}\left(\int_{x\left(\xi_{2}, t_{0}\right)}^{x\left(\xi_{1}, \tau\right)} \frac{F(\eta)}{v\left(\xi_{1}, \eta\right)} d \eta+\frac{\Delta x_{0}}{v\left(\xi_{1}, t_{0}\right)} F\left(x\left(\xi_{1}, t_{0}\right)\right)\right)
\end{aligned}
$$

and

$$
\begin{aligned}
\frac{1}{m} \int_{t_{0}}^{\tau} F\left(\xi_{2}, \theta\right) d \tau \approx \frac{1}{m}\left(\int_{t_{0}}^{\tau-\varepsilon} F\left(\xi_{2}, \theta\right) d \theta+\varepsilon F\left(\xi_{2}, \tau\right)\right) & = \\
& =\frac{1}{m}\left(\int_{x\left(\xi_{2}, t_{0}\right)}^{x\left(\xi_{1}, \tau\right)} \frac{F(\eta)}{v\left(\xi_{2}, \eta\right)} d \eta+\frac{\Delta x_{0}}{v\left(\xi_{1}, t_{0}\right)} F\left(x\left(\xi_{2}, \tau\right)\right)\right) .
\end{aligned}
$$

Thus, for $\Delta x$ we obtain

$$
\begin{aligned}
\Delta x=(1 & \left.+\frac{1}{m v\left(\xi_{1}, t_{0}\right)}\left(F(x)_{x=x\left(\xi_{2}, t\right)} \varepsilon-F(x)_{x=\xi_{1}}\left(t-t_{0}\right)\right)\right) \Delta x_{0}+ \\
& +\int_{t_{0}}^{t}\left(v\left(\xi_{2}, t_{0}\right)-v\left(\xi_{1}, t_{0}\right)+\frac{1}{m} \int_{x\left(\xi_{2}, t_{0}\right)}^{x\left(\xi_{1}, \tau\right)}\left(\frac{F(\eta)}{v\left(\xi_{2}, \eta\right)}-\frac{F(\eta)}{v\left(\xi_{1}, \eta\right)}\right) d \eta\right) d \tau
\end{aligned}
$$

To satisfy condition (4) it is necessary that

$$
\lim _{\Delta x_{0} \rightarrow 0}\left(\left(v_{2}\left(t_{0}\right)-v_{1}\left(t_{0}\right)\right)=0\right.
$$

\footnotetext{
${ }^{4}$ These are concentrated forces which act on a matter point as a whole quantum object. This does not result in infinite acceleration, since, as supposed above, the inertia of each material point is characterized by the mass of the entire object.
} 
(velocity field continuity), and

$$
v\left(\xi_{1}, \eta\right) \equiv v\left(\xi_{2}, \eta\right)
$$

(velocity field stationarity). For the last identity to be satisfied with the conditions under consideration, it is necessary and sufficient to have

$$
v\left(\xi_{2}, \tau\right)=v\left(\xi_{1}, \tau+\varepsilon\right)=v\left(\xi_{1}, \tau\right)+\frac{F}{m} \frac{\Delta x(\tau)}{v\left(\xi_{1}, \tau\right)} .
$$

As $\Delta x \rightarrow 0$, the last expression takes the form

$$
\left(m v \frac{\partial v}{\partial x}+\frac{\partial U(x)}{\partial x}\right) d x=\frac{\partial E}{\partial x} d x=0,
$$

where $E$ is the total mechanical energy of the material point. This means that the material points of the matter field involved in the formation of the physical continuum have the same energy.

Consider the one-dimensional motion of the matter field described above. The infinitesimal individual body having the volume $d \xi$ at time $t=0$ and identified by the material coordinate $\xi=x(0)$ generates the density measure field at time $t$ in the form

$$
\rho^{E}(x, t)=\int_{-\infty}^{\infty} \delta\left(x-x\left(\xi^{E}, t\right)\right) d m,
$$

where $d m=\rho^{E}(\xi) d \xi$ is the measure of the matter particles set occupying the volume $d \xi$ at point in time $t_{0} ; \delta\left(x-x^{E}(\xi, t)\right)$ is the Dirac's $\delta$-function; $x^{E}(\xi, t)$ is the the least action path of a material particle having the energy $E$. This expression can be considered as a continuous equation, since together with the condition (4) it guarantees the conservation of the measure in the form

$$
\rho^{E}(\xi, t) d x(\xi, t)=\rho^{E}\left(\xi, t_{0}\right) d \xi=\rho^{E}\left(\xi, t_{0}\right) d x\left(\xi, t_{0}\right),
$$

where $d x(\xi, t)$ denote the volume occupying a particular set of the material particles at point of time $t$. In accordance with $(2)$ the complex density $\mu^{E}(\xi, t)$ expressed in terms of the density measure as follows

$$
\mu^{E}(\xi, t)=\rho^{E}(\xi, t) \exp \frac{i}{\hbar} S\left[x^{E}\left(\xi, t, t^{p}\right)\right]
$$

The actions in the last expression depend on arbitrary point in time in the past $t^{p}$, which cancel in the continuity equation in the form (5). Then for the continuity equation in term of the complex density $\mu(x, t)$, we get

$$
\begin{array}{r}
\mu^{E}(x, t)=\int_{-\infty}^{\infty} \mu^{E}\left(x_{0}, t_{0}\right) \exp \frac{i}{\hbar}\left(S^{E}\left(x, t, t^{p}\right)-S^{E}\left(x, t_{0}, t^{p}\right)\right) \delta\left(x-x^{E}\left(x_{0}, t\right)\right) d x_{0}= \\
=\int_{-\infty}^{\infty} \mu^{E}\left(x_{0}, t_{0}\right) \exp \frac{i}{\hbar} S^{E}\left(x, t, t_{0}\right) \delta\left(x-x^{E}\left(x_{0}, t\right)\right) d x_{0}
\end{array}
$$


where $x_{0}=x^{E}\left(\xi, t_{0}\right)=\xi$ and for the complex density of an arbitrary physical continuum, as a set of the matter fields, we obtain

$$
\mu(x, t)=\sum_{\alpha} \int_{0}^{\infty} g_{\alpha}(E)\left(\int_{-\infty}^{\infty} \mu^{E}\left(x_{0}, t_{0}\right) \exp \frac{i}{\hbar} S^{E}\left(x, t, t_{0}\right) \delta\left(x-x^{E}\left(x_{0}, t\right)\right) d x_{0}\right) d E
$$

If ambient conditions do not change with time, then the velocity fields corresponding to the matter fields forming a homogeneous (non-compound in terms of [7]) object are stationary. Therefore the field of Lagrangian is stationary too, and the field of the action $S^{E}(x, t)$ has the form

$$
S^{E}(x, t)=L^{E}(x) t+S^{E}\left(x, t_{0}\right) .
$$

Then for the continuity equation for the field of the complex density $\mu_{t}^{E}(x)$, we obtain

$$
\mu^{E}(x, t)=\int_{-\infty}^{\infty} \exp \left(\frac{i}{\hbar} L^{E}(x)\left(t-t_{0}\right)\right) \delta\left(x-x^{E}\left(x_{0}, t\right)\right) \mu^{E}\left(x_{0}, t_{0}\right) d x_{0}
$$

and

$\mu(x, t)=\int_{0}^{\infty} g_{\alpha}(E)\left(\int_{-\infty}^{\infty} \exp \left(\frac{i}{\hbar} L^{E}(x)\left(t-t_{0}\right)\right) \delta\left(x-x^{E}\left(x_{0}, t\right)\right) \mu^{E}\left(x_{0}, t_{0}\right) d x_{0}\right) d E$,

The equation (7) describes the evolution of the complex density $\mu(x, t)$ of a homogeneous object under stationary ambient conditions.

\section{The wave function}

The complex density $\mu$ was introduced regardless of the measurement process, and the classical quantities characterizing material points (and, therefore, determining the function $\mu$ ) are not observables. The wave function is determined by observables generated during the special measurement process. On other hands, both the complex density and the wave function, in accordance with the assumption made, describe the same spatial distribution of the physical continuum. This means that the complex density is a more general quantity and it should display itself as a wave function in the measurement process.

According to the paper [6] the measurement process consists of two stages. On the first of them, the quantum object interacts with the active elements of the apparatus. As the result of this interaction, the energy of the active element increases up to a threshold value. Then a macroscopic registering process is initiated, and spatial distribution of the continuous medium instantly changes, what is formally expressed in the collapse of the wave function. Since the wave function of the of the system reveal itself at the first stage, we consider this stage only.

Let the system consisting of the object and one of the active element be closed (such a consideration is possible if the interaction energies of the active elements each others anothers are negligible compared with their interaction energies with the object). Then the interaction of the object with the active element must obey the equation (6). This equation will be used to determine the dependence of the energy transfer rate from the object to the active particle on the complex density. 
Let $x$ be the position of the object. In general the active element consist of a set of elementary particles. Since, the size of an element is assume to be small compared to the spatial size $\Delta x \sim \rho \partial x / \partial \rho$, the active element can be considered as a quantum particle, whose position we denote by $X^{5}$. In the considered case, the active element is a composite continuous medium described by the function $\mu(X)$, which differs from zero inside a much smaller spatial volume than the function $\mu(x)$.

Let the active element be a harmonic oscillator. By $U(X)$ denote corresponding potential energy. By $U^{i n t}(x, X) \approx U^{i n t}(x, \bar{X})$ (here $\bar{X}$ a mean value of the active element position) denote the interaction energy of the object with the active element. We cannot use the equation (7) because of time dependence of the interaction energy $U^{i n t}(x-\bar{X})$, which depends on the position of the moving material points of the object. We have a non-homogeneous system. The equation (6) is usable in this case. It takes the form

$$
\begin{aligned}
\mu(x, X, t)=\int G\left(E^{a}\right)\left(\int \exp \frac{i}{\hbar} S^{E^{a}}(X,\right. & \left.t, t_{0}\right) I[\bar{X}, \mu(x, \tau), t] \mu_{t_{0}}^{E^{a}}\left(X, t_{0}\right) \times \\
& \left.\times \delta\left(X-X^{E^{a}}\left(X_{0}, \tau\right)\right) d X_{0}\right) d E^{a},
\end{aligned}
$$

where $E^{a}$ is the energy of the active element matter field; $G\left(E^{a}\right)$ is the complex weight of the fields forming the continuous medium of the active element; $X^{E^{a}}\left(X_{0}, \tau\right)$ is the least action path of the active element material point having the energy $E^{a} ; S^{E^{o}}(X, t)=S^{E^{o}}\left[X^{E^{a}}\left(X_{0}, \tau\right), X, t\right]$ is the action on the paths of the active element, excluding the action part corresponding to interaction with the object. The functional

$$
\begin{aligned}
I[\bar{X}, \mu(x, \tau), t]=\int_{0}^{\infty} g\left(E^{o}\right)\left(\int_{-\infty}^{\infty}\right. & \exp \left(\frac{i}{\hbar} \int_{t_{0}}^{t}\left(L^{E^{o}}(x)-U^{i n t}(x-\bar{X})\right) d \tau\right) \times \\
& \left.\times \delta\left(x-x^{E^{o}}\left(x_{0}, \tau\right)\right) \mu^{E^{o}}\left(x_{0}, t_{0}\right) d x_{0}\right) d E^{o}
\end{aligned}
$$

describes the effect of the object on the active element; $E^{o}$ is the total mechanical energy of the object material point; $L^{E^{o}}(x)$ is the spatial field of the object's Lagrangian; $g\left(E^{o}\right)$ is the complex weights of the matter fields of the object.

By $\varepsilon=\left(t-t_{0}\right) / N$ denote an infinitesimal time interval $(N$ is an infinitely large integer); by $t_{k}=t_{0}+k \varepsilon$ denote the digital time variable. By $R$ denote interaction radius of the object with the active element and suppose that the interaction energy $U^{i n t}$ differs from zero only for the range of positions of the object from $\bar{X}-R$ to $\bar{X}+R$. If the time integral on the right hand side of (9) is represented as an integral sum, then for the matter field having energy $E^{o}$ we obtain

$$
I\left[X, \mu^{E^{o}}(x, \tau), t\right]=\int_{-\infty}^{\infty} \cdots \int_{-\infty}^{\infty} \mu^{E^{o}}\left(x_{0}, t_{0}\right) \times
$$

\footnotetext{
${ }^{5}$ The condition limiting the size of the interaction field with the active element, expressed through a change in the density $\rho$ is a considerably less strict than the similar condition expressed through a change in the complex density of $\mu$. The last condition, practically, cannot be realised.
} 


$$
\times \prod_{j=0}^{N} \exp \left(\frac{i}{\hbar} L_{j}\left(x_{j}\right) \varepsilon\right) \exp \left(-\frac{i}{\hbar} U_{j}^{i n t}\left(x_{j}\right) \varepsilon\right) \delta\left(x_{j+1}-x_{j}\right) d x_{j},
$$

Expand the exponents containing the interaction energy in a Taylor series. Holding the first-order term, we get

$$
\begin{aligned}
I\left[X, \mu^{E^{o}}(x, \tau), t\right]=\int_{-\infty}^{\infty} \cdots & \int_{-\infty}^{\infty} \mu_{0}^{E^{o}}\left(x_{0}\right) \times \\
& \times \prod_{j=0}^{N} \mathrm{~T}_{j}\left(x_{j}\right)\left(1-\frac{i}{\hbar} U_{j}^{i n t}\left(x_{j}\right) \varepsilon\right) \delta\left(x_{j+1}-x_{j}\right) d x_{j},
\end{aligned}
$$

where $\mu_{0}^{E^{o}}\left(x_{0}\right) \equiv \mu^{E^{o}}\left(x_{0}, t_{0}\right) ; \mathrm{T}_{j}\left(x_{j}\right)=\exp \frac{i}{\hbar} L_{j}\left(x_{j}\right) \varepsilon$.

Taking into account that

$$
\mu_{j}\left(x_{j}\right) \approx \prod_{k=0}^{j} \mathrm{~T}_{k}\left(x_{k}\right) \mu_{0}^{E^{o}}\left(x_{0}\right),
$$

the last equation takes the form

$$
\begin{aligned}
& I\left[X, \mu_{j}^{E^{o}}(x), N\right]= \\
& =\int_{-\infty}^{\infty} \cdots \int_{-\infty}^{\infty} \prod_{j=0}^{N} \mu_{j}^{E^{o}}\left(x_{j}\right)\left(1-\frac{i}{\hbar} U_{j}^{i n t}\left(x_{j}\right) \varepsilon\right) \delta\left(x_{j+1}-x_{j}\right) d x_{j} \approx \\
& \quad \approx \mu_{N}^{E_{o}}\left(x_{N}\right) \exp \left(-\frac{i}{\hbar} \sum_{j=0}^{N} \int_{\bar{X}-R}^{\bar{X}+R} \mu_{j}^{E_{o}} U^{i n t}\left(\bar{X}-x_{j}\right) d x_{j}\right) .
\end{aligned}
$$

Integrating over all energies $E^{o}$ for $\varepsilon \rightarrow 0$, we obtain

$$
I[X, \mu(x, \tau), t]=\mu^{o}(x, t) \exp \left(-\frac{i}{\hbar} \int_{t_{0}}^{t}\left(\int_{\bar{X}-R}^{\bar{X}-R} \mu^{o}(x, \tau) U^{i n t}(\bar{X}-x) d x\right) d \tau\right) .
$$

Thus, according to (8), the complex density of the continuum of the active particle varies with time as

$$
\begin{aligned}
& \mu^{a}(X, t)=\int_{0}^{\infty} G\left(E^{a}\right)\left(\int _ { - \infty } ^ { \infty } \operatorname { e x p } \frac { i } { \hbar } \left[\int_{t_{0}}^{t}(T(\dot{X})-U(X)-\right.\right. \\
&\left.\left.-\int_{\bar{X}-R}^{\bar{X}-R} \mu^{o}(x, \tau) U^{i n t}(x-\bar{X}) d x\right) d t\right] \times \\
& \quad \times \mu^{\left.E^{a}\left(X_{0}, t_{0}\right) \delta\left(X-X_{0}\right) d X_{0}\right) d E^{a} .}
\end{aligned}
$$

From the classical point of view, the last term of the action in this equation is generated by the the work of the force with which the object acts on the active particle, having the position $\bar{X}$. For this force we have 


$$
\begin{aligned}
& F(\bar{X}, \tau)=\frac{\partial}{\partial \zeta} \int_{-R}^{R} \mu^{o}(\bar{X}+\zeta, \tau) U^{i n t}(\zeta) d \zeta= \\
& \left.=\left(\mu^{o}(\bar{X}+R, \tau)-\mu^{o}(\bar{X}-R), \tau\right)\right) U(R) \approx \\
& \approx \rho^{o}(\bar{X})\left(\exp \frac{i}{\hbar} S^{o}(\bar{X}+R)-\exp \frac{i}{\hbar} S^{o}(\bar{X}-R)\right) U(R) \approx \\
& \approx \rho^{o}(\bar{X}) \exp \frac{i}{\hbar}\left(p^{o}(\bar{X}) \bar{X}-E^{o} \tau\right)\left(\exp \frac{i}{\hbar} p^{o}(\bar{X}) R-\exp \left(-\frac{i}{\hbar} p^{o}(\bar{X}) R\right)\right) U(R)= \\
& =2 i U(R) \sin \left(\frac{2 \pi R}{\lambda(\bar{X})}\right) \mu(\bar{X}, \tau),
\end{aligned}
$$

where $\zeta=x-\bar{X} ; \rho^{o}, S^{o}, p^{o}, E^{o}$ are respectively the density, action, momentum and energy of the object (it is supposed, that the momentum $p$ does not depend on the position in the interaction region); $\lambda(\bar{X})=h / p(\bar{X})$.

Let the active particle is a harmonic oscillator having the cyclic eigenfrequency $\omega_{0}$. Material points of the oscillator move in accordance with the dynamic equation in the form

$$
\ddot{X}+\omega_{0}^{2} X=\frac{1}{m} F(\bar{X}, \tau) .
$$

In accordance with $(10)$ the forces $F(\bar{X}, \tau)$ are the same for all matter points of the active particle continuum and does not depend on the coordinate $X$. Then the energy received by a harmonic oscillator under the action of an external variable force is determined by the expression [8]

$$
E\left(\omega_{0}\right)=\frac{1}{2 m}\left|\int_{-\infty}^{\infty} F(\tau) \exp i \omega_{0} \tau d \tau\right|^{2}
$$

Taking into account (10), we get

$$
E\left(\omega_{0}\right)=\frac{1}{2 m}\left|2 i U(R) \sin \left(\frac{2 \pi R}{\lambda(\bar{X})}\right)\right|^{2}\left|\int_{-\infty}^{\infty} \mu(\bar{X}, \tau) \exp i \omega_{0} \tau d \tau\right|^{2}
$$

Since the duration of the measurement process is less than the time $T$, when the complex density field is nonzero at the observation point, the energy E should be limited not by the time of the force (as in [8]), but by the interaction time $\Delta t=t-t_{0}$. Then the the last expression must be rewritten in the form

$$
E\left(t, t_{0}, \omega_{0}\right)=\beta\left|\int_{-\infty}^{\infty} h(t-\tau) h\left(\tau-t_{0}\right) \mu(\bar{X}, \tau) \exp i \omega_{0} \tau d \tau\right|^{2},
$$

where $h(t-\tau), h\left(\tau-t_{0}\right)$ are Heaviside functions; $t_{0}, t$ are the moments of the beginning and end of the interaction; $\beta$ is a constant characterizing the sensitivity of the active particle. The time interval $\Delta t$ must be large enough so that

$$
\int_{-\infty}^{\infty} h(t-\tau) h\left(\tau-t_{0}\right) \mu(\bar{X}, \tau) \exp i \omega_{0} \tau d \tau \approx \lim _{T \rightarrow \infty} \frac{\Delta t}{T} \int_{-\infty}^{\infty} \mu(\bar{X}, \tau) \exp i \omega_{0} \tau d \tau,
$$


where $T$ is the time when the field of the function $|\mu(\bar{X}, \tau)|$ does not depends on time. Then, for the rate of increase of an active particle energy, we have

$$
\frac{\Delta E}{\Delta t}=\frac{\beta}{T}\left|\int_{-\infty}^{\infty} \mu(\bar{X}, \tau) \exp \left(-i \omega_{0} \tau\right) d \tau\right|^{2}
$$

The completion stage of the measurement process is the macroscopic registering process [6], which is initiated when the energy one of the active particles increases up to a threshold value.The values of the initiation threshold for the different active particles have a statistical straggling (as well as the sensitivity $\beta$ ). Macroscopic changes are initiated by only one active particle, for which the threshold is exceeded before the others. By $E_{n}^{t h}$ denote the threshold energy value for the the active element $n$. This is a random quantity. Then, the random time $t_{n}$ of the "triggering" of the active particle $n$ is determined by the condition

$$
\frac{\beta_{n}}{T}\left|\int_{-\infty}^{\infty} \mu\left(\overline{X_{n}}, \tau\right) \exp \left(-i \omega_{0} \tau\right) d \tau\right|^{2} t_{n}=E_{n}^{t h}
$$

Thus, the registration process is initiated in that active particle for which the value of

$$
\frac{1}{T} \frac{\beta_{n}}{E_{n}^{t h}}\left|\int_{-\infty}^{\infty} \mu\left(\overline{X_{n}}, \tau\right) \exp \left(-i \omega_{0} \tau\right) d \tau\right|^{2}
$$

is maximum. The coefficient before the module in the last expression has a random value from 0 to $\infty$ with the probability density function $f\left(\frac{\beta_{n}}{E_{n}^{t h}}\right)$, which does not depend on the positions of active particles. This coefficient converts the measure into the registration probability $P_{n}$ by the particle $n$ having the eigenfrequency $\omega_{0}$. This probability has the form

$$
C\left|\int_{-\infty}^{\infty} \mu\left(\overline{X_{n}}, \tau\right) \exp \left(-i \omega_{0} \tau\right) d \tau\right|^{2}
$$

where $C$ is a normalization constant. Then, integrating over all $\omega_{0}$, for the interaction radius $R \rightarrow 0$ and $N \rightarrow \infty$, the registration probability density $\rho^{p}(X)^{6}$ takes the form

$$
\rho^{p}(X) \sim\left|\int_{0}^{\infty}\left(\int_{-\infty}^{\infty} \mu(X, \tau) \exp i \omega_{0} \tau d \tau\right) d \omega_{0}\right|^{2}
$$

If to follow to the Born interpretation of the wave function, then we should suppose that

$$
\rho^{p}(X)=\left|\Psi_{\tau}(X)\right|^{2} \sim\left|\int_{0}^{\infty}\left(\int_{-\infty}^{\infty} \mu(X, \tau) \exp i \omega_{0} \tau d \tau\right) d \omega_{0}\right|^{2}
$$

\footnotetext{
${ }^{6}$ However, there is a remark: the limit $R \rightarrow 0$ is incorrect physically for $R \ll \lambda \bar{X}$, because, in this case, the force (9) becomes zero; thus, we suppose that the Born interpretation has the corresponding spatial limit.
} 
This equality will be satisfied if the wave function is the superposition of the harmonic components of the force (10). Supposing this and taking into account that

$$
\Psi_{\tau}(X)=\int_{0}^{\infty} \psi^{\epsilon}(X) \exp \left(-\frac{i}{\hbar} \epsilon \tau\right) d \epsilon,
$$

where $\epsilon$ is a observable energy $\left(\hbar \omega_{0}=\epsilon\right)$, we obtain

$$
\psi^{\epsilon}(X)=\int_{-\infty}^{\infty} \mu(x, \tau) \exp \frac{i}{\hbar} \epsilon \tau d \tau .
$$

Using the expression for the complex density (3), we have

$$
\psi^{\epsilon}(X)=\int_{0}^{\infty}\left(\int_{0}^{\infty} \sum_{\alpha} g_{\alpha}(E) \mu_{\alpha}^{E}(x, \tau) d E\right) \exp \frac{i}{\hbar} \epsilon \tau d \tau .
$$

For infinite one-dimensional motion, the parameter $\alpha$ has a unique value. In this case, the spectral density of the complex measure $\mu^{E}(x, \tau)$ is a travelling wave, that is, $E=\epsilon$, and it is not quantized. In a finite one-dimensional motion, two waves travelling in opposite directions form a stationary wave only for the discrete energy values $E_{n}$. In this case, the superposition of travelling waves $\mu^{E}$ with energies close to $E_{n}$ also contributes to the stationary wave function $\psi^{\epsilon}(X)$, so that $\psi^{E_{n}} \neq \mu^{E_{n}}$.

The proposed representation of the wave function (11) is based on the equality (12) and is not unique. To confirm this representation, we derive the quantum evolution law from the equation (6).

\section{The wave equation}

The continuity equation for the complex density according to eqrefeq:math:ex8 has the form

$$
\mu_{t}(x)=\int_{0}^{\infty}\left(\int_{-\infty}^{\infty} K_{t, t_{0}}^{E}\left(x, x_{0}\right) \mu_{t_{0}}^{E}\left(x_{0}\right) d x_{0}\right) d E
$$

where for the kernel of the integral operator $K_{t, t_{0}}^{E}\left(x, x_{0}\right)$, we have

$$
K_{t, t_{0}}^{E}\left(x, x_{0}\right)=\exp \frac{i}{\hbar} S_{t, t_{0}}^{E}\left(x, x_{0}\right) \delta\left(x-x\left(\xi^{E}, t\right)\right) .
$$

The wave function is expressed in terms of the complex density $\mu_{\tau}(x)$ as follows

$$
\begin{aligned}
& \Psi_{t}(X)=\int_{0}^{\infty}\left(\int_{-\infty}^{\infty} \mu_{t}(x) \exp \frac{i}{\hbar} \epsilon t d t\right) \exp \left(-\frac{i}{\hbar} \epsilon t\right) d \epsilon= \\
= & \int_{0}^{\infty}\left(\int_{-\infty}^{\infty}\left(\int_{0}^{\infty}\left(\int_{-\infty}^{\infty} K_{t, t_{0}}^{E}\left(x, x_{0}\right) \mu_{t_{0}}^{E}\left(x_{0}\right) d x_{0}\right) d E\right) \exp \frac{i}{\hbar} \epsilon t d t\right) \exp \left(-\frac{i}{\hbar} \epsilon t\right) d \epsilon .
\end{aligned}
$$

For fixed final time, initial and final positions, $t_{0}$ is different for material fields corresponding to different energies and motion directions. Then, changing the 
order of integration with respect to the position $x_{0}$ and the energy $E$ in the last expression, we obtain the convolution function $(K * \mu)(t)$. Using the convolution theorem, we finally obtain

$$
\Psi_{t}(x)=\int_{-\infty}^{\infty} K_{t, t_{0}}\left(x, x_{0}\right) \Psi_{t_{0}}\left(x_{0}\right) d x_{0},
$$

where

$$
K_{t, t_{0}}\left(x, x_{0}\right)=\int_{0}^{\infty} \exp \frac{i}{\hbar} S_{t, t_{0}}^{E}(x) \delta\left(x-x\left(\xi^{E}, t\right)\right) d E .
$$

In accordance with [5] this expression is equivalent to the path integral [9]

$$
K_{t, t_{0}}\left(x, x_{0}\right)=\int \exp \left(\frac{i}{\hbar} S\left[x_{0}(\tau)\right]\right)\left[d x_{0}(\tau)\right] .
$$

Thus we obtained the integral wave equation with the kernel in the form of a path integral. For infinitesimal time intervals this equation takes the form of Schrödinger equation [10,11]. Schrödinger steady-state equation is the direct consequence of the fact that the stationary wave function is factorized. This formal mathematical reason for quantization has a physical basis in the observability (in the sense of the possibility of macroscopic registration) of the physical continuum.

\section{Conclusion}

The approach to the description of the phenomena of the microcosm considered here is fundamentally different from the approach of conventional quantum mechanics. First of all is that the quantum mechanics assertion ". . all occurrences of an atomic and molecular order of magnitude, obey the "discontinuous" laws of quanta" [7] is correct only for directly observable processes. Directly observable processes are those that can be detected at any stage by a macroscopic apparatus. As have been shone, this requirement implies a harmonic time dependence of the complex density at the detector location. In the case of finite motion, this, in turn, means that only such mechanical motion can be detected that generates a standing wave of complex density, which result in the quantization of energy. I.e. a superposition of only a stationary states is detectable. But it is not means that other mechanical states do not exist. Thus, there is no reason to consider quantum as a fundamental object and the principle of continuity "natura non facit saltus" is correct for microscopic phenomena.

If complex density waves is generated by the mechanical motion of physical continua definitely, then the set of the matter fields corresponding to the different energies of this motion has the cardinality of continuum. Like energy quantization, countability of the wave functions set is the result of the observability requirement.

Since the waves of complex density are generated by the movement of material fields, the latter can exist even if the superposition of these waves (the complex density $\mu$ of the continuous medium) is equal to zero and the concept of physical vacuum acquires a concrete mathematical image. In turn, the Fourier components of complex density $\mu^{E}$ becomes a mathematical image corresponding to the material waves in a physical vacuum. In accordance with [6] such a possibility is indirectly confirmed by the interaction of particles in entangled states.

Thus all main quantum mechanics phenomena can be described using the simple mechanical model based on the motion of a peculiar continuous medium. 
Moreover, such a representation avoids the problems associated with causality principle, non-epistemic nature of quantum probability, contradiction with the special relativity and so on. This approach is based on the local reality principle.

However, the theory under consideration is not reduced to quantum mechanics representation. It can describe phenomena, that cannot be described by the methods of conventional quantum mechanics. These are, first of all, phenomena connected with the processes are "masked" by uncertainty principle. Really, subject to the description of micro-phenomena by the proposed method of mechanical motion of material fields, we proceed to a deterministic mechanical description and thereby avoid the quantum principle of uncertainty.

Another type of problem that can be solved by the theory under consideration is a description on the fundamental level of the dynamics of open quantum systems [6].

Competing interests. I declare that I have no competing interests.

Author's Responsibilities. I take full responsibility for submitting the final manuscript in print. I approved the final version of the manuscript.

\section{References}

1. Einstein A., Podolsky B., Rosen N. Can quantum mechanics description of physical reality be considered complete?, Phys. Rev., 1935, vol. 47, no. 10, pp. 777-780. doi: 10.1103/PhysRev. 47.777.

2. Schrödinger E. Der stetige Übergang von der Mikro- zur Makromechanik, Naturwissenschaften, 1926, vol. 14, no. 28, pp. 664-666. doi: 10.1007/BF01507634; eng. transl.: Schrödinger E. The continuous transition from micro- to macro mechanics, In: Collected papers on wave mechanics. New York, Chelsea Publishing Co., 1982, pp. 41-44.

3. Bell J. S. Against 'measurement', In: Speakable and Unspeakable in Quantum Mechanics: Collected Papers on Quantum Philosophy. Oxford, Oxford Univ. Press, 2004, pp. 213-231. doi : 10.1017/CB09780511815676.025.

4. Maudlin T. What Bell did, J. Phys. A: Math. Theor., 2014, vol. 47, no. 42, 424010. doi: 10 . 1088/1751-8113/47/42/424010.

5. Samarin A. Yu. Quantum particle motion in physical space, Adv. Studies Theor. Phys., 2014, vol. 8, no. 1, pp. 27-34, arXiv: 1407.3559 [quant-ph]. doi: 10.12988/astp.2014.311136.

6. Samarin A. Yu. Nonlinear dynamics of open quantum systems, Vestn. Samar. Gos. Tekhn. Univ., Ser. Fiz.-Mat. Nauki [J. Samara State Tech. Univ., Ser. Phys. Math. Sci.], 2018, vol. 22, no. 2, pp. 214-224. doi: 10.14498/vsgtu1582.

7. von Neumann J. Mathematische Grundlagen der Quantenmechanik, Grundlehren der mathematischen Wissenschaften, vol. 38. Berlin, Heidelberg, New York, Springer, 1996, ix +262 pp. doi: 10.1007/978-3-642-61409-5.

8. Landau L. D., Lifshitz E. M. Mechanics, vol.1, Course of Theoretical Physics. Oxford, Pergamon Press, 1969, vii+165 pp., https://archive.org/details/Mechanics_541.

9. Feynman R. P. Space-time approach to non-relativistic quantum mechanics, Rev. Mod. Phys., 1948, vol.20, no. 2, pp. 367-387. doi: 10.1103/RevModPhys.20.367.

10. Feynman R. P., Hibbs A. R. Quantum Mechanics and Path Integrals. New York, McGrawHill, 1965.

11. Kac M. Probability and related topics in physical sciences, Lectures in Applied Mathematics. Proceedings of the Summer Seminar (Boulder, Colo., 1957), vol.1. Lonodon, New York, Interscience Publ., 1959, xiii +266 pp. 
Вестн. Сам. гос. техн. ун-та. Сер. Физ.-мат. науки. 2020. Т. 24, № 1. С. 7-21 ISSN: 2310-7081 (online), 1991-8615 (print)

УДК 517.958:530.145

\title{
Механическое движение специфической сплошной среды как физическая основа квантовой эволюции
}

\section{А. Ю. Самарин}

Самарский государственный технический университет, Россия, 443100, Самара, ул. Молодогвардейская, 244.

\begin{abstract}
Аннотация
Квантовая частица рассматривается как сплошная среда, обладающая рядом специфических свойств. Эти свойства сформулированы так, чтобы основные постулаты традиционной квантовой механики были прямым следствием механического движения такой сплошной среды. Представлено детерминистическое описание процесса взаимодействия квантовой частицы с измерительным прибором при измерении координаты. Показана природа возникновения случайности в процессе измерения и выведено правило Борна для пространственной плотности вероятности. Волновая функция интерпретируется как специфическая объемная сила, с которой сплошная среда квантового объекта воздействует на измеритель, а квантовое волновое уравнение выводится из уравнения непрерывности для этой среды. Предложенный подход к представлению микроявлений позволяет исключить ограничения, связанные с принципом неопределённости, и описывать динамику процессов недоступных для рассмотрения методами квантовой механики.
\end{abstract}

Ключевые слова: детерминистическое описание квантовых явлений, сплошная среда, принцип локального реализма, материальное поле, уравнение непрерывности, реалистическое представление волновой функции, правило Борна, принцип неопределенности.

Получение: 21 июля 2019 г. / Исправление: 12 октября 2019 г. Принятие: 11 ноября 2019 г. / Публикация онлайн: 7 февраля 2020 г.

Конкурирующие интересы. Я заявляю, что у меня нет конкурирующих интересов в отношении данной статьи.

Авторская ответственность. Я несу полную ответственность за представление окончательной рукописи в печатном виде. Я одобрил окончательный вариант рукописи.

\section{Научная статья}

(2) (9) Контент публикуется на условиях лицензии Creative Commons Attribution 4.0 International (https://creativecommons.org/licenses/by/4.0/deed.ru)

\section{Образец для цитирования}

$\mathrm{S}$ a mar in A. Y u. Quantum evolution as a usual mechanical motion of peculiar continua, Vestn. Samar. Gos. Tekhn. Univ., Ser. Fiz.-Mat. Nauki [J. Samara State Tech. Univ., Ser. Phys. Math. Sci.], 2020, vol. 24, no. 1, pp. 7-21. doi:10.14498/vsgtu1724.

\section{Сведения об авторе}

Алексей Юръевич Самарин (D) https://orcid.org/0000-0001-7640-3875

кандидат физико-математических наук, доцент; каф. общей физики и физики нефтегазового производства; e-mail: samarin. ay@yahoo.com 